\title{
Evaluation of ultrasonographic optic nerve sheath diameter and central retinal artery Doppler indices by point-of-care ultrasound in pediatric patients with increased intracranial pressure
}

\author{
Nagehan Aslan ${ }^{1 \oplus}$, Dinçer Yıldızdaş ${ }^{1 \oplus}$, Özden Özgür $\mathrm{Horoz}^{1 \oplus}$, Mazhar Özsoy ${ }^{2 \oplus}$, \\ Ahmet Yöntem $^{1 \oplus}$, Eralp Çetinalp ${ }^{2 \oplus}$, Gülen Gül Mert ${ }^{30}$ \\ Departments of ${ }^{1}$ Pediatric Intensive Care, ${ }^{2}$ Neurosurgery and ${ }^{3}$ Pediatric Neurology, Çukurova University Faculty of Medicine, Adana, \\ Turkey.
}

\begin{abstract}
Background. Measurement of the optic nerve sheath diameter (ONSD) with point-of-care ultrasound (POCUS) is a non-invasive and radiation-free technique that can be used to assess increased intracranial pressure (ICP). Ophthalmic artery and central retinal artery Doppler indices can be used like transcranial Doppler to evaluate increased ICP. This study aims to examine the diagnostic value of ONSD measurements and central retinal artery Doppler indices in the evaluation of pediatric patients with increased ICP.
\end{abstract}

Methods. This was a prospective, case-controlled single center study. The study group was comprised of a total of 38 pediatric patients with increased ICP and the control group included 19 healthy children. Ophthalmic ultrasound was performed and ONSD and central retinal artery Doppler indices were measured.

Results. The mean age of the study group was $80.84 \pm 65.12$ months. The mean ONSD was $5.9 \pm 0.8$ (3.6-8.1) $\mathrm{mm}$ in the study group and the mean resistive index (RI) was $0.71 \pm 0.08$ (min:0,55-max:1) and was significantly greater than the control group $(\mathrm{p}<0.001$ and $\mathrm{p}<0.001$, respectively). In terms of predicting increased ICP, the ONSD measurement was the strongest parameter, with its area under the curve: 0.767 (95 percent confidence interval: $0.68-0.85$ ). In the study group, the cut-off value for ONSD was $5.8 \mathrm{~mm}$ (66 percent sensitivity, 100 percent specificity) and the cut-off value for RI was 0.68 (63 percent sensitivity, 83 percent specificity).

Conclusions. Point-of-care ultrasound is a noninvasive and important tool in pediatric intensive care units. Our study is significant as one of the few pediatric studies where central retinal artery Doppler indices are evaluated in addition to OSND, in patients with increased ICP.

Key words: central retinal artery, Doppler indices, optic nerve sheath diameter, point-of-care ultrasound, pediatric, resistive index.

Increased intracranial pressure (ICP) can emerge as a result of many different neurological conditions, such as trauma, infection and intracranial mass. ${ }^{1}$ Early diagnosis and treatment of increased ICP in critically

$\triangle$ Nagehan Aslan

nagehan_aslan@hotmail.com

Received 13th March 2020, revised 21st March 2020, 16th June 2020, accepted 2nd July 2020.

The study was presented at the $63 \mathrm{r}^{\mathrm{d}}$ Turkish National Pediatric Congress, Turkish Republic of Northern Cyprus. ill pediatric patients receiving treatment in pediatric intensive care units (PICU) is crucial for the prevention of neurological damage and mortality. ${ }^{2}$ In the evaluation of increased ICP, the gold standard is the measurement of pressure using a catheter placed in the brain parenchyma or ventricle. ${ }^{3}$ However, this method is invasive, difficult to implement and carries a risk of various complications, such as bleeding and infection; therefore, performing this method is not always possible and new, non-invasive diagnosis methods are emerging., ${ }^{4,5}$ 
The methods of cranial computed tomography (CT) and magnetic resonance imaging (MRI) are frequently used; however, the requirement of sedation, difficulties in the transportation of hemodynamically unstable intensive care patients and radiation exposure of CT are limitations of these methods. ${ }^{6}$

In recent years, the use of point-of-care ultrasound (POCUS) by non-radiologist clinicians has become widespread. ${ }^{7}$ For specialists working in pediatric emergency departments and PICUs, POCUS has become widely used for physical examinations due to the rapid results it offers for critically ill patients. ${ }^{8}$ Ultrasonographic measurement of ONSD is especially important for the clinical follow-up of patients with increased ICP. ${ }^{9}$ The fact that an increase is detected in ONSD in the early phase in case of increased ICP due to its direct relation with subarachnoid distance has been revealed in many studies. ${ }^{10,11}$

The effect of increased ICP on ophthalmic veins is not completely understood. Ophthalmic artery and central retinal artery Doppler indices can now be used in addition to the transcranial Doppler to evaluate increased ICP. ${ }^{12}$ With ultrasonography, the central retinal and ophthalmic arteries can be readily visualized deep in the orbital cavity, in the area where they cross the optic nerve. ${ }^{13}$ Peak systolic velocity (PSV) and end diastolic velocity (EDV) measurements are performed in colored Doppler images. Resistive index (RI) is used to measure resistance against the arterial bloodstream and is calculated using an ultrasound device through a formula with colored Doppler measurements [PSV-EDV)/ PSV $] \cdot{ }^{14}$

This study aims to examine the diagnostic value of ONSD measurements and central retinal artery Doppler indices in the evaluation of PICU patients with increased ICP and to draw attention to the increased usage rate, popularity and importance of the use of POCUS by pediatric intensivists.

\section{Material and Methods}

\section{Patients}

A total of 38 pediatric patients, who were treated in our tertiary PICU and for whom increased ICP was suspected clinically (change in consciousness level, dilated or nonreactive pupil, loss of brain stem reflexes, injury of cranial nerve, Cushing triad) or radiologically (shift, deletion in the sulcus, ventricular collapse, cistern pressure) were prospectively included in this single-center study, over eight months. In addition to the study group, 19 children, who applied to the hospital's general pediatric outpatient clinic for different reasons and who did not show symptoms of increased ICP, were included in the study as the control group. Because the Doppler ultrasound is an extremely difficult method, to eliminate measurement error, low dose sedative drugs were administered to the PICU patients in the study group. For the control group, nonpharmacological sedation methods were used, such as non-nutritive suckling or the use of breast milk or glucose as sedatives for infant subjects. For subjects able to communicate, the method required for obtaining accurate ultrasonographic measurements was explained. Three measurements were taken from each patient in the study group to control for measurement error and the mean value of these measurements were recorded for the last analysis. Similarly, two measurements were taken from each child in the control group and the mean value of these measurements were recorded for the last analysis. The mean value of each child's ONSD and central retinal artery Doppler indices were recorded. Following a radiology expert's evaluation of image quality, 88 measurements from the study group and 23 measurements from the control group were selected for analysis.

The study was conducted following the ethical criteria of the 1964 Helsinki Declaration and was approved by a Çukurova University Faculty of Medicine clinical research ethics committee (Date: January 4, 2019; Meeting number: 84). 
Written informed consent was obtained from the families of the patients.

\section{Ophthalmic ultrasound method}

All ophthalmic ultrasonographic measurements were performed by an experienced pediatric intensive care fellow (N.A.) who completed a POCUS course provided by the Turkish Pediatric Emergency and Intensive Care Society and performed at least 300 ophthalmic ultrasound measurements before the study. The Mindray ultrasound device (Resona7, Mindray Bio-Medical Electronics Co., Ltd., China) 5.1-12.5 MHz linear probe was used for the measurements. The patients were placed in the supine position by raising their heads by 20-30 degrees. The ultrasound system controls acoustic output so as not to exceed a mechanical index (MI) level of 1.9, a spatial peak, temporal average intensities (ISPTA.3) of $50 \mathrm{~mW} / \mathrm{cm}^{2}$ or a thermal index (TI) value of 1.0. ${ }^{15,16}$ After applying sterile gel at room temperature, the ultrasound probe was placed on the right superior eyelid of the patient's closed eye and the ONSD measurement was performed in B mode, $3 \mathrm{~mm}$ behind the optic disc (Fig. 1). First, the central retinal artery was identified in the optic nerve using color Doppler sonography. Afterward PSV and EDV measurements were performed on the saved colored Doppler images (Fig. 2). By using these measurements, RI was

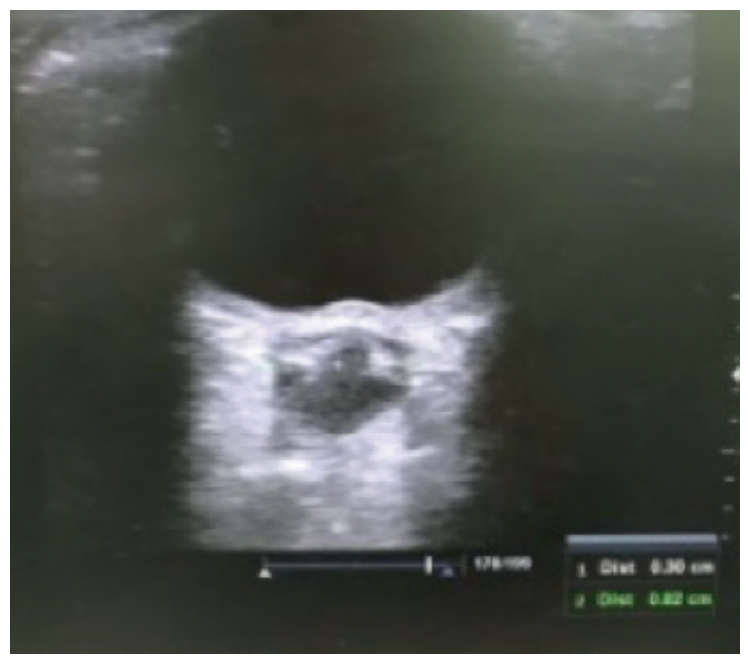

Fig. 1. Ultrasonographic OSND measurement. automatically calculated by the ultrasound device.

\section{Statistical analysis}

The IBM SPSS Statistics Version 20.0 packaged program was used for the statistical analysis of the data. The categorical measurements were summarized in numbers and percentages and numerical measurements were summarized as mean and standard deviation (median and minimum-maximum when necessary). The Chi-square test statistic was used to compare the categorical measurements between the groups. The Mann-Whitney U test was used to compare the numerical measurements between two groups that did not show normal distribution. ROC analysis was performed to detect the most effective parameter in terms of foreseeing increased ICP and to determine the appropriate breakpoints of ONSD. A statistical significance level of 0.05 was implemented for all tests.

\section{Results}

In this study, 88 ophthalmic ultrasound measurements were analyzed on 38 (14 females, 24 males) patients with increased ICP and 23 measurements were analyzed on 19 (nine females, 10 males) patients from the healthy control group. When the underlying causes of increased ICP in the study group were

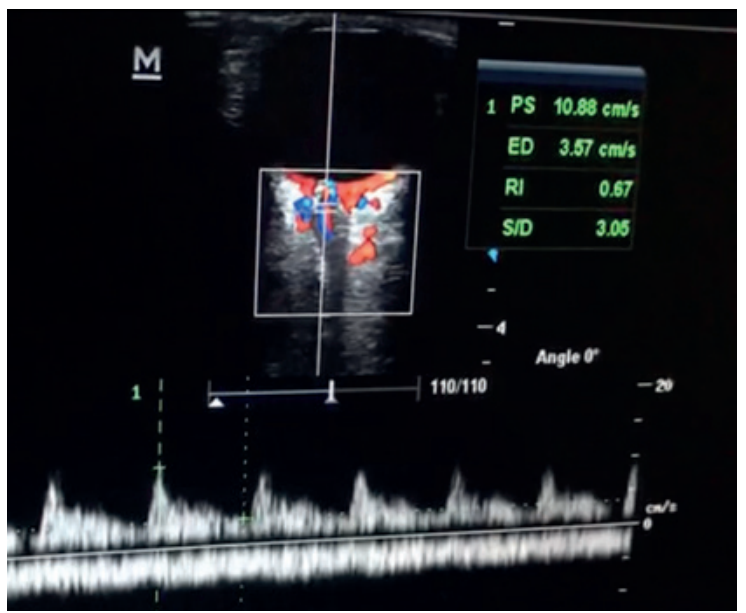

Fig. 2. Measurement of ophthalmic artery Doppler indices. 
examined, it was apparent that nine $(23.7 \%)$ patients had traumatic causes and 29 (76.3 $\%)$ patients had non-traumatic causes. The underlying diagnoses of the patients are given in Table I. The mean age was $88.47 \pm 64.98$ (min: 3-max: 204) months in the whole group. The mean age was $80.84 \pm 65.12$ months in the study group and $97.74 \pm 64.95$ months in the control group. No significant difference was found between the patient and control groups in terms of age and gender $(\mathrm{p}=0.397$ and $\mathrm{p}=0.313$, respectively). While the mean ONSD was $5.9 \pm$ $0.8(3.6-8.1) \mathrm{mm}$ in the study group, the mean RI was $0.71 \pm 0.08$ (min:0,55-max:1) and was significantly greater than the control group $(\mathrm{p}<0.001$ and $\mathrm{p}<0.001$, respectively) (Table II). In terms of predicting increased ICP, the ONSD measurement was determined as the strongest parameter, with its area under the curve: 0.767 (95 percent confidence interval: 0.68-0.85) (Fig. 3). In the study group, the cut-off value

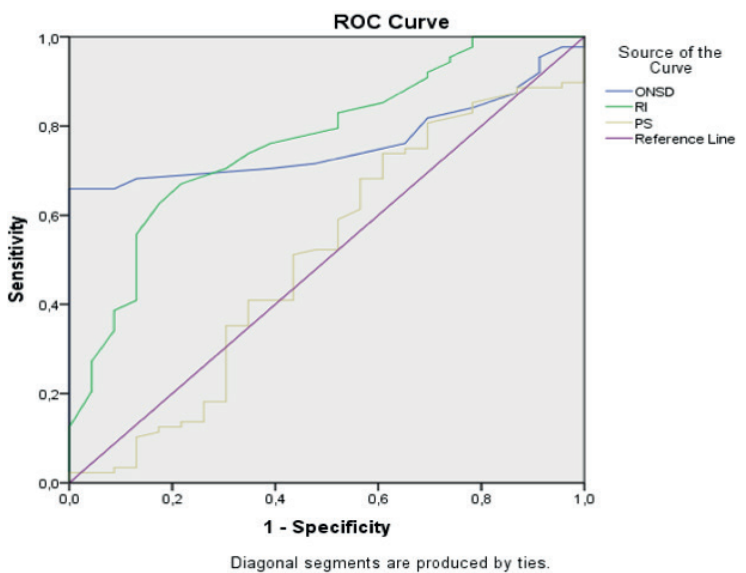

Fig. 3. The ROC curve for factors which predict increased ICP.

Table I. Underlying causes of increased ICP in the patient group.

\begin{tabular}{ll}
\hline Underlying causes of patients & Number $(\%)$ \\
\hline Traumatic causes & $9(23.7)$ \\
Nontraumatic causes & $29(76.3)$ \\
Meningoencephalitis & $5(17.3)$ \\
Intracranial mass & $9(31)$ \\
Postarrest care & $3(10.3)$ \\
Hydrocephalus- ventriculoperitoneal shunt dysfunction & $6(20.7)$ \\
Other & $6(20.7)$ \\
\hline
\end{tabular}

Table II. Comparison of ultrasonographic measurements of the patient and control groups.

\begin{tabular}{lccl}
\hline & Patient group & Control group & \\
N=38, number of & N=19, number of & \\
measurements=88) & measurements:23) & \\
Mean \pm SD & Mean \pm SD & Median & \\
& Median & (min-max) & \\
& $($ min-max $)$ & $5.2 \pm 0.3$ & $<0.001$ \\
ONSD $(\mathrm{mm})$ & $5.9 \pm 0.8$ & $5.3(4.4-5.7)$ & \\
& $6.1(3.6-8.1)$ & $0.62 \pm 0.07$ & $<0.001$ \\
Resistive index & $0.71 \pm 0.08$ & $16.59 \pm 9.03$ & \\
& $0.71(0.55-1)$ & $13.39(7.94-36.97)$ & 0.925 \\
Peak systolic velocity & $16.02 \pm 8.05$ & $6.01 \pm 3.06$ & \\
& $13.98(6.91-55.92)$ & $5.66(2.83-12.76)$ & 0.017 \\
End diastolic velocity & $4.3 \pm 1.86$ & &
\end{tabular}

ONSD: optic nerve sheath diameter 
for ONSD was determined as $5.8 \mathrm{~mm}$ with 66 percent sensitivity and 100 percent specificity and the cut-off value for RI was determined as 0.68 with 63 percent sensitivity and 83 percent specificity.

\section{Discussion}

POCUS is a very important method for intensivists and its usage has become widespread since it offers the opportunity to make cost-effective, non-invasive, radiationfree and repeated measurements of ONSD. ${ }^{12,17}$ Evaluation of myocardial contraction and cardiac index by critical care echocardiography, rapid diagnosis and treatment of pneumothorax by lung ultrasound, detection of pleural fluid and drainage by ultrasound, decreasing radiation exposure in the radiological followup of pneumonia by substituting for chest radiography, clinical follow-up of the patient with increased ICP by measuring the ONSD by ophthalmic ultrasound, determination of the fluid around the liver, spleen and kidney with rapid abdominal evaluation (and, if necessary, drainage of the fluid by ultrasound-guided paracentesis), placement of central venous catheter by ultrasound, evaluation of the volume status of the patient by measuring vena cava inferior diameters and guiding the fluid treatment to be applied are among the usage areas of POCUS by pediatric intensivists..$^{9,13,18-21}$

ONSD has an ongoing character with dura mater, arachnoid mater and pia mater. ${ }^{18}$ When ICP increases, it is directly reflected on ONSD and an increase may be detected even before the development of papillary edema. ${ }^{14,22}$ As a rapid and noninvasive method, the ultrasonographic ONSD measurement has become a new tool in monitoring elevated ICP for pediatricians in emergency and intensive care departments. ${ }^{11}$ The usage of ocular ultrasound for the indirect assessment of ICP by measuring ONSD is already well known. ${ }^{9}$ POCUS, which is distinguished from other diagnosis methods for being fast, easy, cheap, repeatable and especially for being non-invasive, is preferred as a new method for the detection of increased ICP and clinical follow-up. ${ }^{22,23}$

Rehman Siddiqui et al. ${ }^{24}$ conducted a pediatric study and reported that the limit for ONSD was $>4 \mathrm{~mm}$ in newborns, $>4.71 \mathrm{~mm}$ in those between 1-10 years of age and $>5.43$ in those above 10 years (sensitivity 100 percent, specificity 60-66.7 percent). In another study, Irazuzta et al. ${ }^{25}$ evaluated 13 patients diagnosed with pseudotumor cerebri syndrome and stated the mean ONSD as $5.5 \pm 1.2 \mathrm{~mm}$ for the right eye and $5.4 \pm 1 \mathrm{~mm}$ for the left eye. In a study evaluating 72 pediatric patients who received invasive ICP catheters, ONSD cut-off value was stated as 5.28 $\mathrm{mm}$ for intracranial pressure $>15 \mathrm{mmHg}$ and $5.57 \mathrm{~mm}$ for intracranial pressure $>20 \mathrm{mmHg}{ }^{26}$ In the current study, the mean ONSD was $5.9 \pm$ $0.8 \mathrm{~mm}$ in the group with suspected increased ICP and $5.2 \pm 0.3 \mathrm{~mm}$ in the control group. Our results showed the cut-off value as $5.8 \mathrm{~mm}$ (sensitivity: 66 percent, specificity: 100 percent) for ONSD in the patient group.

In the literature, the exact effect of increased ICP on ophthalmic veins is not well known. ${ }^{23}$ In a study with adult subjects, Ebraheim et al. ${ }^{27}$ looked at colored Doppler ophthalmic ultrasound on 24 adult patients diagnosed with pseudotumor cerebri syndrome and did not detect a significant difference to the control group measurements. On the contrary, Karami et al. ${ }^{28}$ performed colored Doppler ophthalmic ultrasonographic measurements on patients diagnosed with increased ICP and noted a significant elevation in the Doppler indices of the patient group. Gura et al..$^{29}$ determined a significant correlation between retinal RI and intracranial pressure measured with a catheter inside the ventricle. In another study by Riggs et al. ${ }^{30}$ that looked at the ophthalmic artery Doppler ultrasonographic measurements conducted on 13 pediatric patients with brain death, a significant decrease was observed in PSV and the RI was determined as one in all patients. In another adult study conducted by Tarzamni et al. ${ }^{23}$ the Doppler measurements of 30 patients with increased ICP and 30 healthy individuals 
in the control group were compared and ONSD, PSV and RI were found to be significantly elevated. In the current study, ONSD and RI were also found to be significantly elevated in the study group and the cut-off value for RI was determined as 0.68 with 63 percent sensitivity and 83 percent specificity.

For ophthalmic diagnostic applications, the US Food and Drug Administration (FDA) has set maximum recommended exposure levels of MI, TI and ISPTA.3. ${ }^{15}$ These are very significant limits to ensure safe evaluation and the protection of the optic globe. As determined by the FDA, there are different maximum limits for different types of ultrasonographic methods. In an article about the safety considerations of ophthalmic ultrasound, Harris G.R. ${ }^{16}$ recommends the safety limits for the use of ophthalmic ultrasound. The FDA's maximum recommended acoustic output level guidelines have been adhered to and the technical characteristics of the device used have been carefully monitored throughout this study.

The major limitation of this study lies in describing increased ICP without using an invasive ICP catheter. The gold standard for measurement of ICP is by a catheter placed in the brain parenchyma or ventricle. However, since this method is invasive and has various complications, it cannot be applied easily. This study opts for the use of clinical and radiological methods to measure an increase in ICP. Unfortunately, our study group is a suspected increased ICP patient group. Another limitation is the number of patients. To compensate for the low number of participants, additional measurements were evaluated. In addition to ONSD, the central retinal artery Doppler indices (RI, PSV, EDV) were measured and compared to the healthy control group's ultrasonographic measurements.

In conclusion; POCUS may become the new stethoscope for pediatric intensive care specialists because of its wide usage area. One of the most important usage areas of POCUS is ophthalmic ultrasound. In follow up for critically ill pediatric patients with increased ICP, ultrasonographic OSND and ophthalmic and central retinal artery RI measurements, which are non-invasive and can be repeated pending clinical necessity, appear promising for the future in terms of the early diagnosis of increased ICP and essential emergency intervention. This research is important because it is one of the few pediatric studies where central retinal artery Doppler indices are evaluated in addition to OSND in patients with increased ICP. There is a need for further studies with larger series to evaluate the acceptance of POCUS as a noninvasive and radiation-free technique for measure increased ICP by the medical community.

\section{REFERENCES}

1. Goldsmith W, Plunkett J. A biomechanical analysis of the causes of traumatic brain injury in infants and children. Am J Forensic Med Pathol 2004; 25: 89-100.

2. Stocchetti N, Maas AIR. Traumatic intracranial hypertension. N Engl J Med 2014; 370: 2121-2130.

3. Robba C, Cardim D, Czosnyka M, et al. Ultrasound non-invasive intracranial pressure assessment in paediatric neurocritical care: a pilot study. Childs Nerv Syst 2020; 36: 117-124.

4. Robba C, Bragazzi NL, Bertuccio A, et al. Effects of prone position and positive end-expiratory pressure on noninvasive estimators of ICP: a pilot study. J Neurosurg Anesthesiol 2017; 29: 243-250.

5. Robba C, Cardim D, Tajsic T, et al. Ultrasound noninvasive measurement of intracranial pressure in neurointensive care: a prospective observational study. PLoS Med 2017; 14: e1002356.

6. Miglioretti DL, Johnson E, Williams A, et al. The use of computed tomography in pediatrics and the associated radiation exposure and estimated cancer risk. JAMA Pediatr 2013; 167: 700-707.

7. Moore CL, Copel JA. Point-of-care ultrasonography. N Engl J Med 2011; 364: 749-757.

8. O’Brien AJ, Brady RM. Point-of-care ultrasound in paediatric emergency medicine. J Paediatr Child Health 2016; 52: 174-180.

9. Dubourg J, Javouhey E, Geeraerts T, Messerer M, Kassai B. Ultrasonography of optic nerve sheath diameter for detection of raised intracranial pressure: a systematic review and meta-analysis. Intensive Care Med 2011; 37: 1059-1068. 
10. Killer HE, Laeng HR, Flammer J, Groscurth P. Architecture of arachnoid trabeculae, pillars, and septa in the subarachnoid space of the human optic nerve: anatomy and clinical considerations. $\mathrm{Br} \mathrm{J}$ Ophthalmol 2003; 87: 777-781.

11. CShirodkar CG, S. Rao SM, Mutkule DP, Harde YR, Venkategowda PM, Mahesh MU. Optic nerve sheath diameter as a marker for evaluation and prognostication of intracranial pressure in Indian patients: an observational study. Indian J Crit Care Med 2014; 18: 728-734.

12. Dubost C, Le Gouez A, Zetlaoui PJ, Benhamou D, Mercier FJ, Geeraerts T. Increase in optic nerve sheath diameter induced by epidural blood patch: a preliminary report. Br J Anaesth 2011; 107: 627-630.

13. Xirouchaki N, Magkanas E, Vaporidi K, et al. Lung ultrasound in critically ill patients: comparison with bedside chest radiography. Intensive Care Med 2011; 37: 1488-1493.

14. Jang T, Aubin C. The use of serial ocular ultrasonography in the care of patients with a head injury. Ann Emerg Med 2005; 45: 336-337.

15. Guidance for Industry and Food and Drug Administration Staff. Information for Manufacturers Seeking Marketing Clearance of Diagnostic Ultrasound Systems and Transducers, September 9, 2008. https://www.fda.gov/downloads/UCM070911. pdf (Accessed on 25 February, 2019).

16. Harris GR. Safety considerations for diagnostic ultrasound in the eye. J Ultrasound Med 2019; 38: 1163-1165.

17. Geeraerts T, Merceron S, Benhamou D, Vigué B, Duranteau J. Non-invasive assessment of intracranial pressure using ocular sonography in neurocritical care patients. Intensive Care Med 2008; 34: 2062-2067.

18. Longjohn M, Wan J, Joshi V, Pershad J. Point-ofcare echocardiography by pediatric emergency physicians. Pediatr Emerg Care 2011; 27: 693-696.

19. Dickman E, Tessaro MO, Arroyo AC, Haines LE, Marshall JP. Clinician-performed abdominal sonography. Eur J Trauma Emerg Surg 2015; 41: 481492.

20. Lamperti M, Bodenham AR, Pittiruti M, et al. International evidence-based recommendations on ultrasound-guided vascular access. Intensive Care Med 2012; 38: 1105-1117.
21. Jauregui J, Nelson D, Choo E, et al. The BUDDY (Bedside Ultrasound to Detect Dehydration in Youth) study. Crit Ultrasound J 2014; 6: 15.

22. Friedman DI, Jacobson DM. Diagnostic criteria for idiopathic intracranial hypertension. Neurology 2002; 59: 1492-1495.

23. Tarzamni MK, Derakhshan B, Meshkini A, et al. The diagnostic performance of ultrasonographic optic nerve sheath diameter and color Doppler indices of the ophthalmic arteries in detecting elevated intracranial pressure. Clin Neurol Neurosurg 2016; 141: 82-88.

24. Rehman Siddiqui NU, Haque A, Abbas Q, Jurair $\mathrm{H}$, Salam B, Sayani R. Ultrasonographic optic nerve sheath diameter measurement for raised intracranial pressure in a tertiary care center of a developing country. J Ayub Med Coll Abbottabad 2018; 30: 495500 .

25. Irazuzta JE, Brown ME, Akhtar J. Bedside optic nerve sheath diameter assessment in the identification of increased intracranial pressure in suspected idiopathic intracranial hypertension. Pediatr Neurol 2016; 54: 35-38.

26. Kerscher SR, Schöni D, Neunhoeffer F, et al. The relation of optic nerve sheath diameter (ONSD) and intracranial pressure (ICP) in pediatric neurosurgery practice - Part II: influence of wakefulness, method of ICP measurement, intra-individual ONSD-ICP correlation and changes after therapy. Childs Nerv Syst 2020; 36: 107-115.

27. Ebraheim AM, Mourad HS, Kishk NA, Badr Eldin N, Saad AA. Sonographic assessment of the optic nerve and ophthalmic vessels in patients with idiopathic intracranial hypertension. Neurol Res 2018; 40: 728735.

28. Karami M, Shirazinejad S, Shaygannejad V, Shirazinejad Z. Transocular Doppler and optic nerve sheath diameter monitoring to detect intracranial hypertension. Adv Biomed Res 2015; 4: 231.

29. Gura M, Silav G, Isik N, Elmaci I. Noninvasive estimation of cerebral perfusion pressure with transcranial Doppler ultrasonography in traumatic brain injury. Turk Neurosurg 2012; 22: 411-415.

30. Riggs BJ, Cohen JS, Shivakumar B, et al. Doppler ultrasonography of the central retinal vessels in children with brain death. Pediatr Crit Care Med 2017; 18: 258-264. 